\title{
The neural mechanism by which fatigue sensation suppresses physical performance: a magnetoencephalography study
}

Takashi Matsuo ( $\square$ matsuo.takashi@med.osaka-cu.ac.jp)

Osaka City University

\section{Akira Ishii}

Osaka City University

\section{Takayuki Minami}

Osaka City University

Hitoshi Nanjo

Osaka City University

\section{Takahiro Yoshikawa}

Osaka City University

\section{Research Article}

Keywords: fatigue sensation, physical performance, inhibition system, magnetoencephalography (MEG)

Posted Date: February 26th, 2021

DOI: https://doi.org/10.21203/rs.3.rs-272798/v1

License: (c) (1) This work is licensed under a Creative Commons Attribution 4.0 International License. Read Full License

Version of Record: A version of this preprint was published at Experimental Brain Research on October 24th, 2021. See the published version at https://doi.org/10.1007/s00221-021-06250-1. 


\section{Abstract}

The right dorsolateral prefrontal cortex (DLPFC) has been proposed to be the brain region regulating performance through fatigue sensation in fatigue, but direct evidence has been lacking for right DLPFC activation when physical performance is suppressed in the presence of fatigue sensation. We examined whether the right DLPFC is activated when physical performance is suppressed by re-experiencing a fatigue sensation. Eighteen healthy male volunteers participated. They performed a rest session followed by a handgrip session to induce physical fatigue sensation. Then, they were instructed to re-experience the state of the right hand with (i.e., the target condition) and without (i.e., the control condition) fatigue sensation as experienced in the handgrip and rest sessions, respectively, while performing motor imagery of maximum handgrip of the right hand. Neural activity during both conditions was recorded using magnetoencephalography. The level of fatigue sensation was higher in the target condition than in the control condition. Decreases of handgrip strength and beta band power in the right Brodmann's area 46 were observed in the target condition, suggesting that the right DLPFC is involved in the regulation of physical performance through fatigue sensation. These findings may help elucidate the neural mechanisms regulating performance under fatigue conditions.

\section{Introduction}

Fatigue is a significant and common problem in modern society ${ }^{1,2}$. More than $20-30 \%$ of the general population in European countries and the United States reportedly complain of substantial fatigue ${ }^{3-7}$ and more than half of the adult population in Japan may experience fatigue ${ }^{8}$. In addition, fatigue-related diseases such as chronic fatigue syndrome reduce quality of life ${ }^{9}$.

Fatigue is defined as a decline in the ability to perform or the efficiency of performing mental and/or physical activities, caused by excessive mental or physical activity or disease, and often accompanied by a peculiar sense of discomfort, a desire to rest, and a decline in motivation, referred to as fatigue sensation ${ }^{10}$. Fatigue sensation works as a biological alarm to encourage rest and thus avoid disrupting homeostasis ${ }^{11,12}$. The fatigue sensation is therefore thought to play an important role in regulating physical and mental performance.

Regulation of performance through fatigue sensation has been proposed to involve the inhibition system related to physical and/or mental fatigue. In the case of physical fatigue, the inhibition system increases inhibitory inputs to the primary motor cortex (M1) to limit the recruitment of motor units and/or slow the firing rate of active motor units in the $\mathrm{M} 1$ to avoid disrupting homeostasis ${ }^{11-13}$. Neural activity related to the inhibition system has been examined using magnetoencephalography (MEG), and the right dorsolateral prefrontal cortex (DLPFC) seems to be involved in the inhibition system: In a study in which a classical conditioning procedure was used to activate the inhibition system related to physical fatigue, neural activity in the right DLPFC was positively associated with the level of fatigue sensation, which was supposed to correlate with activity of the inhibition system ${ }^{14}$. However, no decrease in physical 
performance (i.e., handgrip strength in this case) caused by activation of the inhibition system was confirmed in the study. Since the relationship between alteration of neural activity in the right DLPFC and decreased physical performance caused by activation of the inhibition system has yet to be determined, assessment of whether the right DLPFC has a role to play in the suppression of physical performance is warranted to clarify the neural mechanisms of the inhibition system related to physical fatigue.

The present study aimed to confirm that the physical fatigue sensation suppresses physical performance and to assess whether activation of the right DLPFC is related to the suppression of physical performance caused by the fatigue sensation. Neural activity while performing motor imagery of maximum handgrip while re-experiencing physical fatigue sensation that had actually been experienced during a prior session was recorded using MEG. Spatial filtering analyses of MEG data were performed to detect changes in oscillatory power that reflected changes in neural dynamics ${ }^{15-17}$.

\section{Methods}

\section{Participants}

Eighteen healthy male volunteers participated in this study. Mean ( \pm standard deviation [SD]) age was $22.1 \pm 1.2$ years. All participants were right-handed according to the Edinburgh Handedness Inventory ${ }^{18}$. Individuals taking chronic medications that affect the central nervous system, individuals with a history of mental or brain and/or upper extremity disorder, and current smokers were excluded. The ethics committee at Osaka City University approved the study protocol (approval number, 4355). Each participant provided written informed consent to participate in this study in accordance with the Declaration of Helsinki and the Ethical Guidelines for Medical and Health Research Involving Human Subjects in Japan (Ministry of Education, Culture, Sports, Science and Technology and Ministry of Health, Labour and Welfare).

\section{Experimental design}

The present study was performed on two separate days (Figure 1A). Intervals between these two experimental days ranged from 3 to 10 days. On the first day, participants performed a rest session, then a handgrip session. On the second day, participants performed an MEG session. In the rest session, participants were instructed to view visual cues presented on a display for $10 \mathrm{~min}$. The visual cue for the rest session was the repeated presentation of a white cross for $1500 \mathrm{~ms}$ followed by presentation of a blue asterisk for $2500 \mathrm{~ms}$. In the handgrip session, participants performed handgrip trials with the right hands using a handgrip device (EXG $30 \mathrm{~kg}$; Alinco, Osaka, Japan) in time with visual cues for the handgrip session. The visual cue for the handgrip session was the repeated presentation of a white cross for $1500 \mathrm{~ms}$ (i.e., corresponding to the rest period) followed by presentation of a green triangle for 2500 ms (i.e., corresponding to the handgrip period). Participants were instructed to grasp the handgrip device at maximum power during the handgrip period ${ }^{19}$. Each participant lay supine on a bed in a magnetically shielded room during both rest and handgrip sessions. 
In the MEG session, participants lay supine on the bed in the magnetically shielded room. The MEG session consisted of two conditions (i.e., a target condition and a control condition). Each condition was performed in a double-crossover fashion and consisted of 150 trials. In the target condition, participants were instructed to re-experience the fatigue sensation of the right hand experienced in the handgrip session during imagery of the maximum grip of the right hand guided by the imaginary handgrip cues. In the control condition, participants were instructed to re-experience the state of their right hand in the absence of the fatigue sensation experienced in the rest session during imagery of the maximum grip of the right hand guided by "imaginary handgrip" cues. These imaginary handgrip cues comprised visual and auditory presentations (Figure 1B). The visual presentation was a white circle projected onto a screen placed in front of the participant using a projector (PG-B10S; Sharp, Osaka, Japan). The auditory presentation comprised two pacing cues (i.e., a 440-Hz beep for $200 \mathrm{~ms}$ as a pacing cue) and an imaginary handgrip cue (i.e., a 1000-Hz beep for $200 \mathrm{~ms}$ as a imaginary handgrip cue). The gaps between the pacing cues and that between the pacing and imaginary handgrip cues were $1000 \mathrm{~ms}$. Participants were asked to maintain motor imagery of maximum grip of the right hand for $2000 \mathrm{~ms}$ from the onset of the imaginary handgrip cue to the onset of the first pacing cue for the next trial in each condition. A 10min rest period was provided between the target and control conditions. Just before and after the rest and handgrip sessions and the target and control conditions, participants were asked to rate their subjective level of physical fatigue using a visual analog scale (VAS) with scores ranging from 0 (minimum fatigue) to 100 (maximum fatigue) and grip strength was assessed using a grip dynamometer (ST-100; Toei Light Co., Saitama, Japan).

\section{MEG recording}

As originally described by Matsuo et al. ${ }^{20}$, MEG was recorded using a 160-channel whole-head-type MEG system (MEG Vision; Yokogawa Electric Corporation, Tokyo, Japan) with a magnetic field resolution of 4 $\mathrm{fT} / \mathrm{Hz}^{1 / 2}$ in the white noise region. Sensor and reference coils were gradiometers with a $15.5-\mathrm{mm}$ diameter and $50-\mathrm{mm}$ baseline, and the two coils were separated by $23 \mathrm{~mm}$. Sampling rate was $1,000 \mathrm{~Hz}$ and the obtained data were high-pass-filtered at $0.3 \mathrm{~Hz}$.

\section{MEG analysis}

Analyses of MEG data were performed using a similar method to that described in a previous study ${ }^{21}$. Magnetic noise from outside the magnetically shielded room was eliminated by subtracting the data obtained from reference coils using MEG 160 software (Yokogawa Electric Corporation, Tokyo, Japan). Epochs of MEG data including artifacts were identified visually and excluded before analysis. Spatial filtering analysis of MEG data was performed to identify changes in oscillatory brain activity reflecting the time-locked cortical activities caused by performing the target and control conditions on the second day.

MEG data were bandpass-filtered at $4-8 \mathrm{~Hz}, 8-13 \mathrm{~Hz}, 13-25 \mathrm{~Hz}$, and $25-58 \mathrm{~Hz}$ using the finite impulse response filtering method implemented in Brain Rhythmic Analysis for MEG software (BRAM; Yokogawa Electric Corporation). After bandpass filtering, the locations and intensities of cortical activities were 
estimated using BRAM, which uses a narrow-band adaptive spatial filtering algorithm ${ }^{22,23}$. Voxel size was set at $5.0 \times 5.0 \times 5.0 \mathrm{~mm}$. Under both target and control conditions, the power of oscillatory brain activity in the temporal window of $500 \mathrm{~ms}$ from $500-2,000 \mathrm{~ms}$ after the onset of presentation of the imaginary handgrip cue was calculated relative to that in the period from -600 to $0 \mathrm{~ms}$ from the onset of presentation of the imaginary handgrip cue (i.e., baseline). MEG data were further analyzed using statistical parametric mapping (SPM8; Wellcome Department of Cognitive Neurology, London, UK) implemented in Matlab (MathWorks, Natick, MA). MEG parameters were transformed into the Montreal Neurological Institute T1-weighted image template. Anatomically normalized MEG data were filtered using a Gaussian kernel of $20 \mathrm{~mm}$ (full-width at half-maximum) in the $x-, y-$, and $z$-axes. Individual MEG data were then incorporated into a random-effects model. These estimated parameters were used to create "contrast" images for group analyses. The significance of differences in oscillatory power in the right BA 46 between conditions was assessed using $t$ statistics (i.e., paired t-test with small volume correction). The threshold for the paired t-test was set at $P<0.0021$ (family-wise error correction for multiple comparisons), considering multiple comparisons among increased or decreased oscillatory power, number of temporal windows (i.e., three temporal windows), and frequencies (i.e., four frequencies). However, the threshold of statistical parametric mapping shown in our figure was set at $P<$ 0.05 (family-wise error correction for multiple comparisons) for the purpose of the presentation. Identification of the brain region (i.e., BA 46) was performed using WFU_PickAtlas version 3.0.4 (http://fmri.wfubmc.edu/software/pickatlas).

In addition, to assess relationships between alterations in neural activity and alterations in physical fatigue sensation caused by re-experiencing the fatigue sensation, contrasts in alteration of oscillatory power corresponding to [(imaginary handgrip/baseline in target condition) - (imaginary handgrip/baseline in control condition)] was created and a one-sample $t$-test with alteration of the physical fatigue sensation (i.e., increased physical fatigue sensation in target condition relative to control condition) as a covariate was performed (i.e., correlation analysis). Correlation analysis was performed in the whole brain and the threshold for analysis was set at $P<0.05$ (family-wise error correction for multiple comparisons).

\section{Overlay of magnetic resonance $(M R)$ images}

As originally described in Ishii et al. ${ }^{21}$, anatomical magnetic resonance imaging (MRI) was performed using a Philips Achieva 3.0 TX scanner (Royal Philips Electronics, Eindhoven, the Netherlands) to permit registration of magnetic source locations with respective anatomical locations. Five markers (Medtronic Surgical Navigation Technologies, Broomfield, CO) were attached to the scalp (i.e., two markers $10 \mathrm{~mm}$ in front of the left and right tragus, one marker $35 \mathrm{~mm}$ above the nasion, and two markers $40 \mathrm{~mm}$ to either side of the marker above the nasion). MEG data were superimposed on MRI images using information obtained from these markers and the MEG localization coils. 
All values are presented as mean $\pm S D$ unless otherwise stated. A paired $t$-test was used to compare levels of handgrip strength and fatigue sensation between before and after target and control conditions and rest and handgrip sessions. Pearson's correlation analysis was performed to assess associations between decreased handgrip strength in the target condition and increased subjective level of physical fatigue sensation in the target condition. All $P$ values were two-tailed, and values $<0.05$ were considered statistically significant for the tests.

\section{Results}

\section{Physical fatigue sensation}

The subjective level of physical fatigue sensation after the rest session was not altered compared with that before the rest session, and that after the handgrip session was increased compared with that before the handgrip session $\left(t_{17}=0.627, P=0.539\right.$ and $t_{17}=4.999, P<0.001$, respectively; paired $t$-test; Figure $2 A)$. The subjective level of physical fatigue sensation after the target condition was increased compared with that before the target condition, and that after the control condition was not altered compared with that before the control condition $\left(t_{17}=2.449, P=0.025\right.$ and $t_{17}=1.968, \mathrm{P}=0.066$, respectively; paired $t$ test; Figure 2B).

\section{Handgrip strength}

Handgrip strength on the first day was analyzed for 17 participants because of failure to save handgrip strength data for 1 participant. Handgrip strength on the second day was analyzed for 18 participants.

Handgrip strength after the rest session was not altered compared with that before the rest session, and that after the handgrip session was decreased compared with that before the handgrip session $\left(\mathrm{t}_{16}=\right.$ $0.474, P=0.679$ and $t_{16}=6.419, P<0.001$, respectively; paired $t$-test; Figure $\left.3 \mathrm{~A}\right)$. Handgrip strength after the target condition was decreased compared with that before the target condition, and that after the control condition was not altered compared with that before the control condition $\left(t_{17}=2.436, P=0.026\right.$ and $\mathrm{t}_{17}=1.903, P=0.963$, respectively; paired $t$-test; Figure 3B). The decrease in handgrip strength under the target condition was greater than that in the control condition $\left(\mathrm{t}_{17}=2.841, P<0.001\right.$, paired $t$-test; Figure $3 \mathrm{C}$ ). In addition, the decreased handgrip strength in the target condition was positively associated with increased subjective levels of physical fatigue sensation in the target condition $(r=0.521, P=0.027$, Figure 4).

\section{Spatial filtering analysis of MEG data}

MEG data from two participants were excluded because the numbers of trials of MEG data after the removal of trials contaminated with artifacts were insufficient for analysis (i.e., MEG data with $<24$ epochs were excluded from our analysis). Decreased beta band power in the right BA 46 in the temporal window of 1,500-2,000 ms after the onset of presentation of the imaginary handgrip cue observed in the target condition was greater than that observed in the control condition (Figure 5; Table 1). In addition, 
correlations were seen between alterations in neural activity during the imaginary handgrip in the target condition relative to those in the control condition and increased subjective levels of physical fatigue sensation in the target condition relative to those in the control condition. Increased fatigue sensation was associated negatively with increased theta band power in BA 22, positively with decreased alpha band power in BA 39, and positively with increased gamma band power in the precentral gyrus (Figure 6; Table 2).

\section{Discussion}

In the present study, participants performed a handgrip session that was intended to induce sensations of physical fatigue on the first day. On the second day, the participants performed the target condition of the MEG session, in which they were instructed to imagine performing maximum grip (i.e., motor imagery of maximum hand grip) by the right hand while re-experiencing the fatigue sensation of the right hand experienced in the handgrip session on the first day. Performing the target condition caused an increase in physical fatigue sensation and a decrease in handgrip strength. The increased subjective level of physical fatigue sensation in the target condition was positively associated with the decreased handgrip strength in the target condition. In the MEG session, the decreased beta band power in the right BA 46 observed under the target condition was greater than that observed under the control condition. In addition, the increased subjective levels of physical fatigue sensation in the target condition relative to that in the control condition were associated with increased theta band power in BA 22, decreased alpha band power in BA 39, and increased gamma band power in the precentral gyrus.

In this study, increased physical fatigue sensation and decreased handgrip strength were observed in the target condition on the second day, in which motor imagery of maximum grip of the right hand was performed while re-experiencing the fatigue sensation of the right hand experienced on the first day. We employed the procedure of imaginary handgrip because actual motion of the hands causes electromagnetic noise that would make the MEG data difficult to analyze. In fact, the neural activity involved in executing an action is reportedly similar to that observed during motor imagery of the action 24,25 and some aspects of the neural mechanisms related to the inhibitory system of fatigue have been successfully investigated using the procedure of imaginary handgrip ${ }^{14,26,27}$. Since the participants selfgenerated fatigue sensation by re-experiencing the fatigue sensation under the target condition of the MEG session and the subjective level of physical fatigue sensation caused in the target condition was positively associated with decreased handgrip strength, self-generation of the fatigue sensation was confirmed to suppress physical performance (i.e., handgrip strength in the present study).

The alteration of neural activity in the right BA 46 (i.e., the DLPFC) caused by the imagery of maximum grip by the right hand while re-experiencing the fatigue sensation in the right hand was assessed using MEG. Decreased beta band power in the right BA 46 was observed in the temporal window of 1,500$2,000 \mathrm{~ms}$ after the onset of the imaginary handgrip in the target condition. Our results indicated that the right DLPFC was involved in the regulation of physical performance through activation of the inhibition system caused by fatigue sensation. Since decreases in alpha and beta band power can be interpreted as 
activation of the cortical area involved in cognitive information processing ${ }^{15}$, the decreased beta band power in the right BA 46 observed in the present study can be interpreted as the right BA 46 being activated to regulate physical performance in the presence of fatigue sensation.

The increased subjective level of physical fatigue sensation was positively associated with increased gamma band power in the precentral gyrus. The precentral gyrus is the anatomical location of the primary motor cortex (M1) and includes a portion of the supplementary motor area (SMA) ${ }^{28-30}$. The M1 and SMA reportedly display the increased gamma band power (i.e., event-related synchronization of the gamma band) just after movement onset, with this increase being sustained for almost the entire movement period ${ }^{31-34}$. The increased gamma band power is thought to indicate sensorimotor integration in the motor cortex, reflecting motor planning ${ }^{35,36}$. In addition, these increases in gamma band power in the M1 and SMA are observed not only during actual motor execution, but also during motor imagery 37 . Taking such findings into consideration, the correlation between gamma band activity in the precentral gyrus and the level of fatigue sensation observed in our study may suggest enhanced adjustment of motor planning in the precentral brain regions caused by the induction of a fatigue sensation to properly suppress motor output and thus maintain homeostasis.

The increased subjective level of physical fatigue sensation was associated with increased theta band power in BA 22 and decreased alpha band power in BA 39. BA 39 is reportedly related to re-experiencing past events ${ }^{38,39}$. BA 22 and BA 39 are also reportedly involved in re-experiencing sensations of physical fatigue ${ }^{40}$. The neural activity in these brain regions may thus be observed in relation to re-experiencing the fatigue sensation generated in this study.

Various limitations to the present study should be considered. First, all participants were healthy males. To generalize our findings, studies with healthy females and participants suffering from fatigue or fatigue-related pathologies in future studies may be of great value. Second, since the level of fatigue sensation perceived is subjective and assessment of the level of fatigue sensation thus relies on selfreports such as the VAS scores used in the present study, the temporal resolution of assessing the level of fatigue sensation was insufficient to follow alterations in level of fatigue sensation within each trial. In our study, decreased beta band power in the right BA 46 was observed in the temporal window of 1,500$2,000 \mathrm{~ms}$ after starting the imaginary handgrip. The activation of the BA 46 in the last 500-ms temporal window in each trial can be speculated to be due to a gradual increase in the level of fatigue sensation from the onset of the imaginary handgrip toward the end of the imaginary handgrip within each trial. The level of fatigue sensation in the last 500-ms temporal window would thus be higher than that in the previous temporal windows. However, since the temporal resolution of assessing the level of fatigue sensation was low, as discussed above, we cannot confirm the interpretation of the finding that the decreased beta band power in the right BA 46 was observed in the temporal window of 1,500-2,000 ms after onset of the imaginary handgrip.

In conclusion, we showed that the fatigue sensation induced by re-experiencing a previously experienced fatigue sensation can decrease physical performance and that activation of the right BA 46 was related 
to the activity of the inhibition system to regulate physical performance in the presence of fatigue sensation. Our findings are expected to contribute to elucidation of a better understanding of the neural mechanisms by which fatigue sensation suppresses physical performance in fatigue and to the development of methods to deal with fatigue and fatigue-related problems.

\section{Declarations}

\section{Acknowledgments}

We wish to thank Forte Science Communications for editorial help with the manuscript and Manryoukai Imaging Clinic for assistance with MRI. We also wish to thank Mr. Kazuki Imoto, Mr. Harutoshi Nakano and Mr. Akiyoshi Miura for help in performing our experiments. This work was supported by JSPS KAKENHI grant no. $16 \mathrm{H} 03248$.

\section{Data availability}

The Ethics Committee of Osaka City University which approved the protocol of our present study does not allow public sharing of the original data. Code for experimental task can be received by e-mail upon reasonable request.

\section{Author contributions}

Takashi Matsuo: Conceptualization, Methodology, Investigation, Formal analysis, Data curation, WritingOriginal Draft, Writing-Review \& Editing; Akira Ishii: Conceptualization, Methodology, Investigation, Formal analysis, Data curation, Writing-Original Draft, Writing-Review \& Editing; Takayuki Minami: Investigation; Hitoshi Nanjo: Investigation; Takahiro Yoshikawa: Supervision. All authors reviewed the manuscript.

\section{References}

1 Caldwell, J. A., Caldwell, J. L., Thompson, L. A. \& Lieberman, H. R. Fatigue and its management in the workplace. Neurosci Biobehav Rev 96, 272-289, doi:10.1016/j.neubiorev.2018.10.024 (2019).

2 Ishii, A. et al. The neural effects of positively and negatively re-experiencing mental fatigue sensation: a magnetoencephalography study. Exp Brain Res 236, 1735-1747, doi:10.1007/s00221-0185260-0 (2018).

3 David, A. et al. Tired, weak, or in need of rest: fatigue among general practice attenders. BMJ 301, 1199-1202 (1990).

4 Pawlikowska, T. et al. Population based study of fatigue and psychological distress. BMJ 308, 763-766 (1994). 
5 Loge, J. H., Ekeberg, O. \& Kaasa, S. Fatigue in the general Norwegian population: normative data and associations. J Psychosom Res 45, 53-65 (1998).

6 Cella, D., Lai, J. S., Chang, C. H., Peterman, A. \& Slavin, M. Fatigue in cancer patients compared with fatigue in the general United States population. Cancer 94, 528-538, doi:10.1002/cncr.10245 (2002).

7 van't Leven, M., Zielhuis, G. A., van der Meer, J. W., Verbeek, A. L. \& Bleijenberg, G. Fatigue and chronic fatigue syndrome-like complaints in the general population. European journal of public health 20, 251-257, doi:10.1093/eurpub/ckp113 (2010).

8 Watanabe, Y. in Fatigue Science for Human Health (eds Y. Watanabe et al.) 5-11 (Springer, 2007).

9 Sotzny, F. et al. Myalgic Encephalomyelitis/Chronic Fatigue Syndrome - Evidence for an autoimmune disease. Autoimmunity Reviews 17, 601-609, doi:10.1016/j.autrev.2018.01.009 (2018).

10 Kitani, T. Term Commitee of Japanese Society of Fatigue Science. Nihon Hirougakkaishi (In Japanese) 6, 1 (2011).

11 Tanaka, M., Ishii, A. \& Watanabe, Y. Neural mechanisms underlying chronic fatigue. Reviews in the neurosciences 24, 617-628, doi:10.1515/revneuro-2013-0035 (2013).

12 Ishii, A., Tanaka, M. \& Watanabe, Y. Neural mechanisms of mental fatigue. Reviews in the neurosciences 25, 469-479, doi:10.1515/revneuro-2014-0028 (2014).

13 Taylor, J. L. \& Gandevia, S. C. A comparison of central aspects of fatigue in submaximal and maximal voluntary contractions. J Appl Physiol (1985) 104, 542-550, doi:10.1152/japplphysiol.01053.2007 (2008).

14 Tanaka, M., Ishii, A. \& Watanabe, Y. Neural mechanism of central inhibition during physical fatigue: a magnetoencephalography study. Brain Res 1537, 117-124, doi:10.1016/j.brainres.2013.08.054 (2013).

15 Pfurtscheller, G. \& Lopes da Silva, F. H. Event-related EEG/MEG synchronization and desynchronization: basic principles. Clin Neurophysiol 110, 1842-1857 (1999).

16 Hillebrand, A., Singh, K. D., Holliday, I. E., Furlong, P. L. \& Barnes, G. R. A new approach to neuroimaging with magnetoencephalography. Hum Brain Mapp 25, 199-211, doi:10.1002/hbm.20102 (2005).

17 David, O., Kilner, J. M. \& Friston, K. J. Mechanisms of evoked and induced responses in MEG/EEG. Neuroimage 31, 1580-1591, doi:10.1016/j.neuroimage.2006.02.034 (2006).

18 Oldfield, R. C. The assessment and analysis of handedness: the Edinburgh inventory. Neuropsychologia 9, 97-113 (1971). 
19 Ishii, A., Tanaka, M. \& Watanabe, Y. The neural mechanisms of re-experiencing physical fatigue sensation: a magnetoencephalography study. Exp Brain Res, doi:10.1007/s00221-016-4648-y (2016).

20 Matsuo, T. et al. Neural effects of hand-grip-activity induced fatigue sensation on appetite: a magnetoencephalography study. Scientific reports 9,11044 , doi:10.1038/s41598-019-47580-z (2019).

21 Ishii, A., Tanaka, M., Yoshikawa, T. \& Watanabe, Y. Evidence for unconscious regulation of performance in fatigue. Scientific reports 7, 16103, doi:10.1038/s41598-017-16439-6 (2017).

22 Dalal, S. S. et al. Five-dimensional neuroimaging: localization of the time-frequency dynamics of cortical activity. Neuroimage 40, 1686-1700, doi:10.1016/j.neuroimage.2008.01.023 (2008).

23 Sekihara, K. \& Nagarajan, S. S. Adaptive Spatial Filters for Electromagnetic Brain Imaging. 1-203 (Springer Verlag, 2008).

24 Decety, J. Do imagined and executed actions share the same neural substrate? Brain Res Cogn Brain Res 3, 87-93, doi:10.1016/0926-6410(95)00033-x (1996).

25 Abbruzzese, G., Avanzino, L., Marchese, R. \& Pelosin, E. Action Observation and Motor Imagery: Innovative Cognitive Tools in the Rehabilitation of Parkinson's Disease. Parkinsons Dis 2015, 124214, doi:10.1155/2015/124214 (2015).

26 Tanaka, M., Ishii, A. \& Watanabe, Y. Neural correlates of central inhibition during physical fatigue. PLoS One 8, e70949, doi:10.1371/journal.pone.0070949 (2013).

27 Tanaka, M., Ishii, A. \& Watanabe, Y. Neural effect of mental fatigue on physical fatigue: a magnetoencephalography study. Brain Res 1542, 49-55 (2014).

28 Ugur, H. C. et al. Arterial vascularization of primary motor cortex (precentral gyrus). Surg Neurol 64 Suppl 2, S48-52, doi:10.1016/j.surneu.2005.07.049 (2005).

29 Banker, L. \& Tadi, P. in StatPearls (StatPearls Publishing Copyright @ 2020, StatPearls Publishing LLC., 2020).

30 Turner, J. A. et al. Application of neuroanatomical ontologies for neuroimaging data annotation. Front Neuroinform 4, doi:10.3389/fninf.2010.00010 (2010).

31 de Lange, F. P., Jensen, O., Bauer, M. \& Toni, I. Interactions between posterior gamma and frontal alpha/beta oscillations during imagined actions. Front Hum Neurosci 2, 7, doi:10.3389/neuro.09.007.2008 (2008).

32 van Wijk, B. C., Beek, P. J. \& Daffertshofer, A. Neural synchrony within the motor system: what have we learned so far? Front Hum Neurosci 6, 252, doi:10.3389/fnhum.2012.00252 (2012). 
33 Crone, N. E., Miglioretti, D. L., Gordon, B. \& Lesser, R. P. Functional mapping of human sensorimotor cortex with electrocorticographic spectral analysis. II. Event-related synchronization in the gamma band. Brain 121 (Pt 12), 2301-2315, doi:10.1093/brain/121.12.2301 (1998).

34 Pfurtscheller, G. Spatiotemporal ERD/ERS patterns during voluntary movement and motor imagery. Supp/ Clin Neurophysio/ 53, 196-198, doi:10.1016/s1567-424x(09)70157-6 (2000).

35 Pfurtscheller, G., Graimann, B., Huggins, J. E., Levine, S. P. \& Schuh, L. A. Spatiotemporal patterns of beta desynchronization and gamma synchronization in corticographic data during self-paced movement. Clinical Neurophysiology 114, 1226-1236, doi:10.1016/s1388-2457(03)00067-1 (2003).

36 Ohara, S. et al. Movement-related change of electrocorticographic activity in human supplementary motor area proper. Brain 123 (Pt 6), 1203-1215, doi:10.1093/brain/123.6.1203 (2000).

37 Amo Usanos, C., Boquete, L., de Santiago, L., Barea Navarro, R. \& Cavaliere, C. Induced GammaBand Activity during Actual and Imaginary Movements: EEG Analysis. Sensors (Basel) 20, doi:10.3390/s20061545 (2020).

38 Denkova, E., Botzung, A., Scheiber, C. \& Manning, L. Implicit emotion during recollection of past events: a nonverbal fMRI study. Brain Res 1078, 143-150, doi:10.1016/j.brainres.2006.01.061 (2006).

39 Botzung, A., Denkova, E. \& Manning, L. Experiencing past and future personal events: functional neuroimaging evidence on the neural bases of mental time travel. Brain and cognition 66, 202-212, doi:10.1016/j.bandc.2007.07.011 (2008).

40 Ishii, A. et al. The neural mechanisms of re-experiencing mental fatigue sensation: a magnetoencephalography study. PLoS One 10, e0122455, doi:10.1371/journal.pone.0122455 (2015).

\section{Tables}

Table 1. Brain regions showing change in oscillatory power between target and control conditions

\begin{tabular}{|c|c|c|c|c|c|c|c|}
\hline \multirow[t]{2}{*}{ Decrease/increase } & \multirow[t]{2}{*}{ Frequency } & \multirow[t]{2}{*}{ Location } & \multirow[t]{2}{*}{ BA } & \multicolumn{3}{|c|}{ MNI coordinates $(\mathrm{mm})$} & \multirow[t]{2}{*}{ Z value } \\
\hline & & & & $x$ & $y$ & z & \\
\hline Decrease & $13-25 \mathrm{~Hz}$ & middle frontal gyrus & 46 & 52 & 43 & 30 & 3.81 \\
\hline
\end{tabular}

BA, Brodmann area; MNI, Montreal Neurological Institute.

$x, y, z$ : Stereotaxic coordinate.

Data were obtained from random-effect analysis (paired $t$-test, $P<0.021$, family-wise error rate). 
Table 2. Brain regions showing correlation with increased subjective levels of physical fatigue sensation between target and control conditions

\begin{tabular}{|c|c|c|c|c|c|c|c|c|}
\hline \multirow[t]{2}{*}{ Decrease/increase } & \multirow[t]{2}{*}{ Frequency } & \multirow[t]{2}{*}{ Location } & \multirow[t]{2}{*}{ Positive/Negative } & \multirow[t]{2}{*}{ BA } & \multicolumn{3}{|c|}{$\begin{array}{l}\text { MNI } \\
\text { coordinates } \\
(\mathrm{mm})\end{array}$} & \multirow[t]{2}{*}{$\begin{array}{l}Z \\
\text { value }\end{array}$} \\
\hline & & & & & $x$ & $y$ & z & \\
\hline Increase & $4-8 \mathrm{~Hz}$ & $\begin{array}{l}\text { Superior } \\
\text { temporal } \\
\text { gyrus }\end{array}$ & Negative & 22 & -68 & -37 & 15 & 4.17 \\
\hline Increase & $25-58 \mathrm{~Hz}$ & $\begin{array}{l}\text { Precentral } \\
\text { gyrus }\end{array}$ & Positive & & 47 & -12 & 35 & 3.8 \\
\hline Decrease & $8-13 \mathrm{~Hz}$ & $\begin{array}{l}\text { Superior } \\
\text { temporal } \\
\text { gyrus }\end{array}$ & Positive & 39 & 42 & -52 & 10 & 4.46 \\
\hline
\end{tabular}

BA, Brodmann area; MNI, Montreal Neurological Institute.

$x, y, z$ : Stereotaxic coordinates.

Data were obtained from random-effects analysis (paired $t$-test, $P<0.05$, family-wise error rate).

\section{Figures}

A

First day
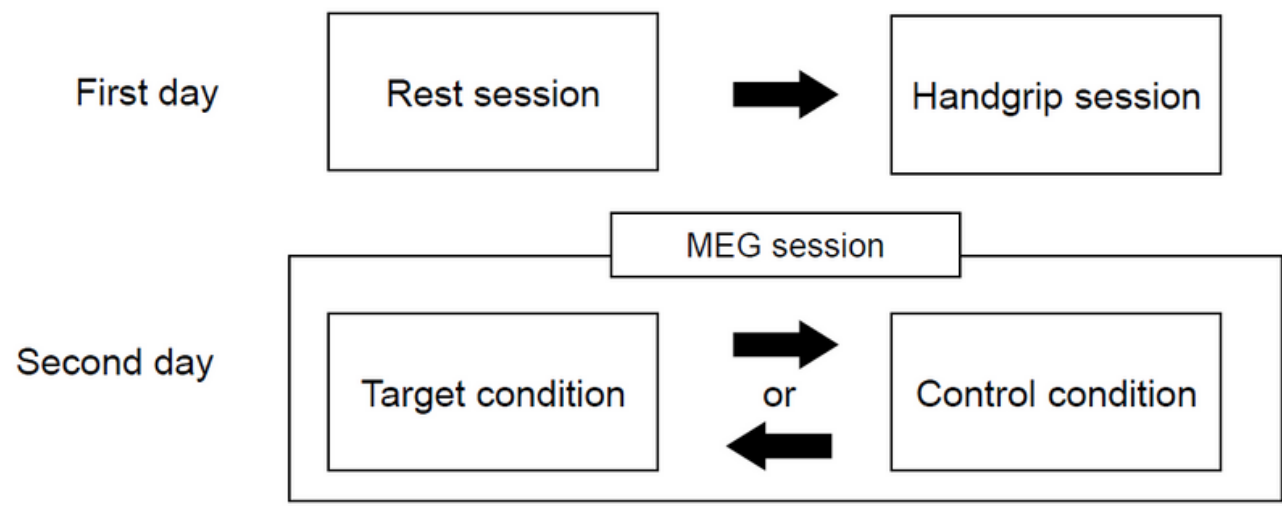

B

1 trial

Next trial Fixation

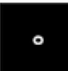

Pacing cue (beep, $440 \mathrm{~Hz}$ )

Pacing cue (beep, $440 \mathrm{~Hz}$ )

Handgrip cue (beep, $1000 \mathrm{~Hz}$ )

Pacing cue (beep, $440 \mathrm{~Hz}$ )
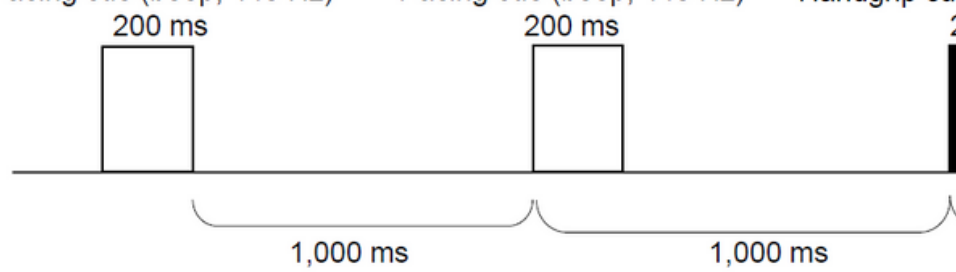

$200 \mathrm{~ms}$

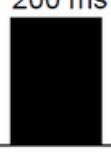

$200 \mathrm{~ms}$ 


\section{Figure 1}

Experimental design A) The study was performed on two separate days. The experiment on the first day consisted of a rest session in which participants were instructed only to view visual cues presented on a display so that they did not experience physical fatigue, and a hand grip session to induce the sensation of physical fatigue. The MEG session on the second day consisted of a target condition and a control condition. In the target condition, participants were instructed to re-experience the sensation of fatigue in the right hand as experienced in the handgrip session, during imagery of maximum grip by the right hand. In the control condition, participants were instructed to re-experience the sensation of no fatigue in the right hand as experienced in the rest session, during imagery of maximum grip by the right hand. B) Imaginary handgrip cues consisted of visual and auditory presentations. The visual presentation was a white circle projected onto a screen. This visual cue was presented over each experiment on the second day. The auditory presentation consisted of two pacing cues (i.e., a 440-Hz beep for $200 \mathrm{~ms}$ as a pacing cue) followed by one imaginary handgrip cue (i.e., a 1000- $\mathrm{Hz}$ beep for $200 \mathrm{~ms}$ as a imaginary handgrip cue).
A
B
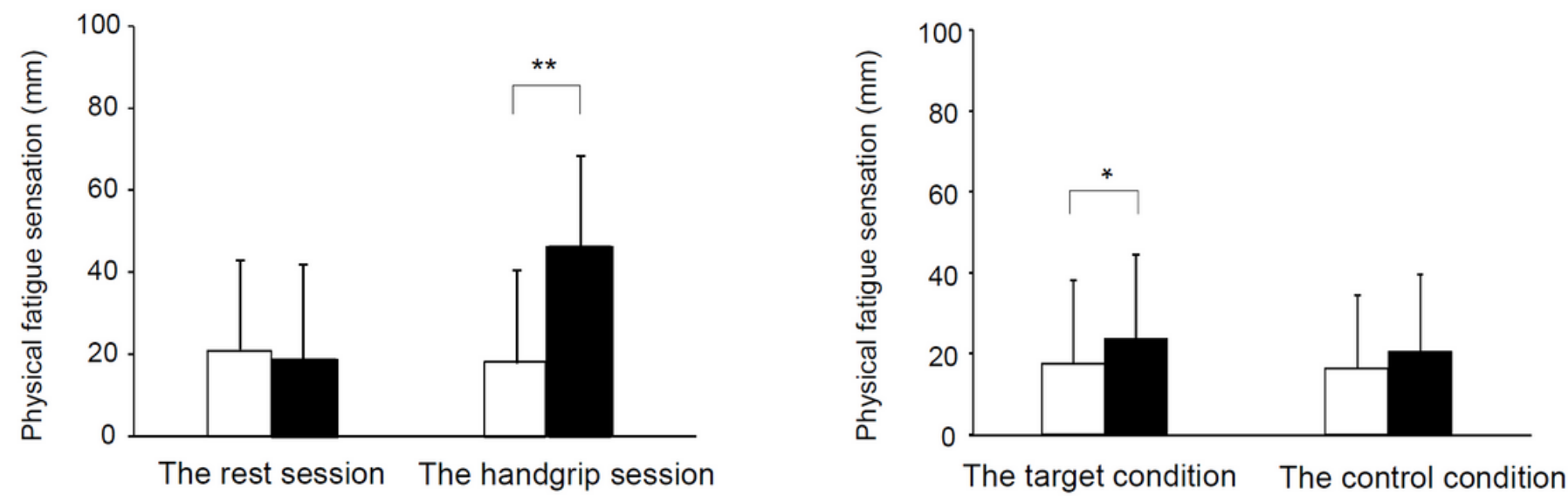

Figure 2

Subjective level of physical fatigue sensation A) Subjective levels of physical fatigue sensation before and after the rest and handgrip sessions on the first day. Open columns indicate values assessed before sessions. Closed columns indicate values assessed after sessions. B) Subjective levels of physical fatigue sensation before and after target and control conditions on the second day. Open columns indicate values assessed before conditions. Closed columns indicate values assessed after conditions. Data are presented as mean $\pm S D$. $* P<0.05$ and $* * P<0.001$, paired t-test. 
A

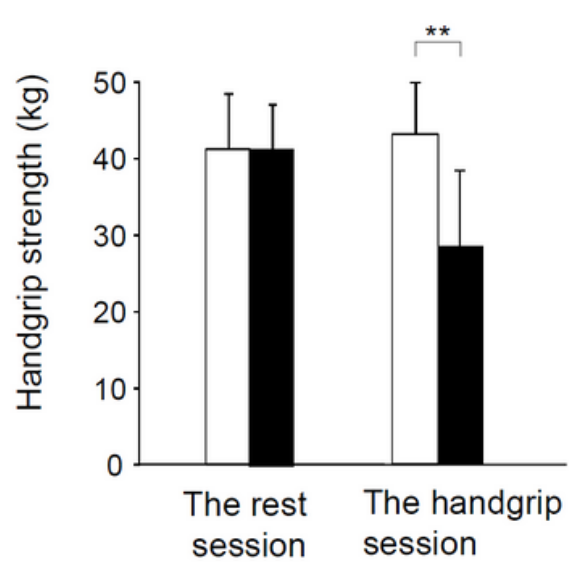

B

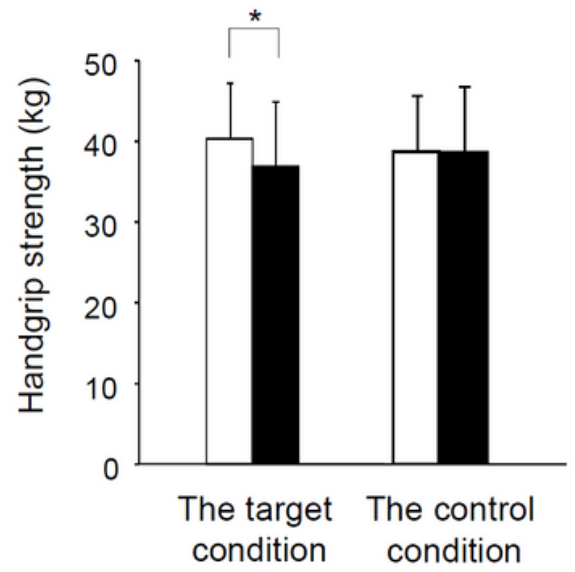

C

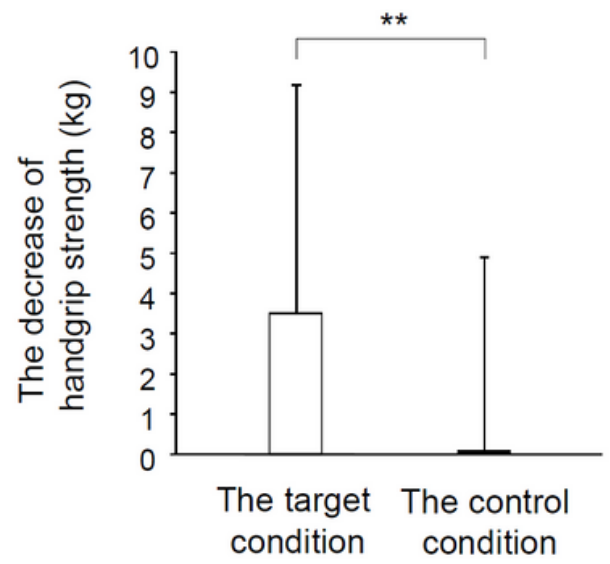

\section{Figure 3}

Handgrip strength A) Handgrip strength assessed before and after rest and handgrip sessions on the first day. Open columns indicate values assessed before sessions. Closed columns indicate values assessed after sessions. B) Handgrip strength assessed before and after target and control conditions on the second day. Open columns indicate values assessed before conditions. Closed columns indicate values assessed after conditions. C) Decrease in handgrip strength in target and control conditions on the second day. Data are presented as mean $\pm S D$. ${ }^{*} P<0.05$ and ${ }^{*} \mathrm{P}<0.001$, paired t-test. 


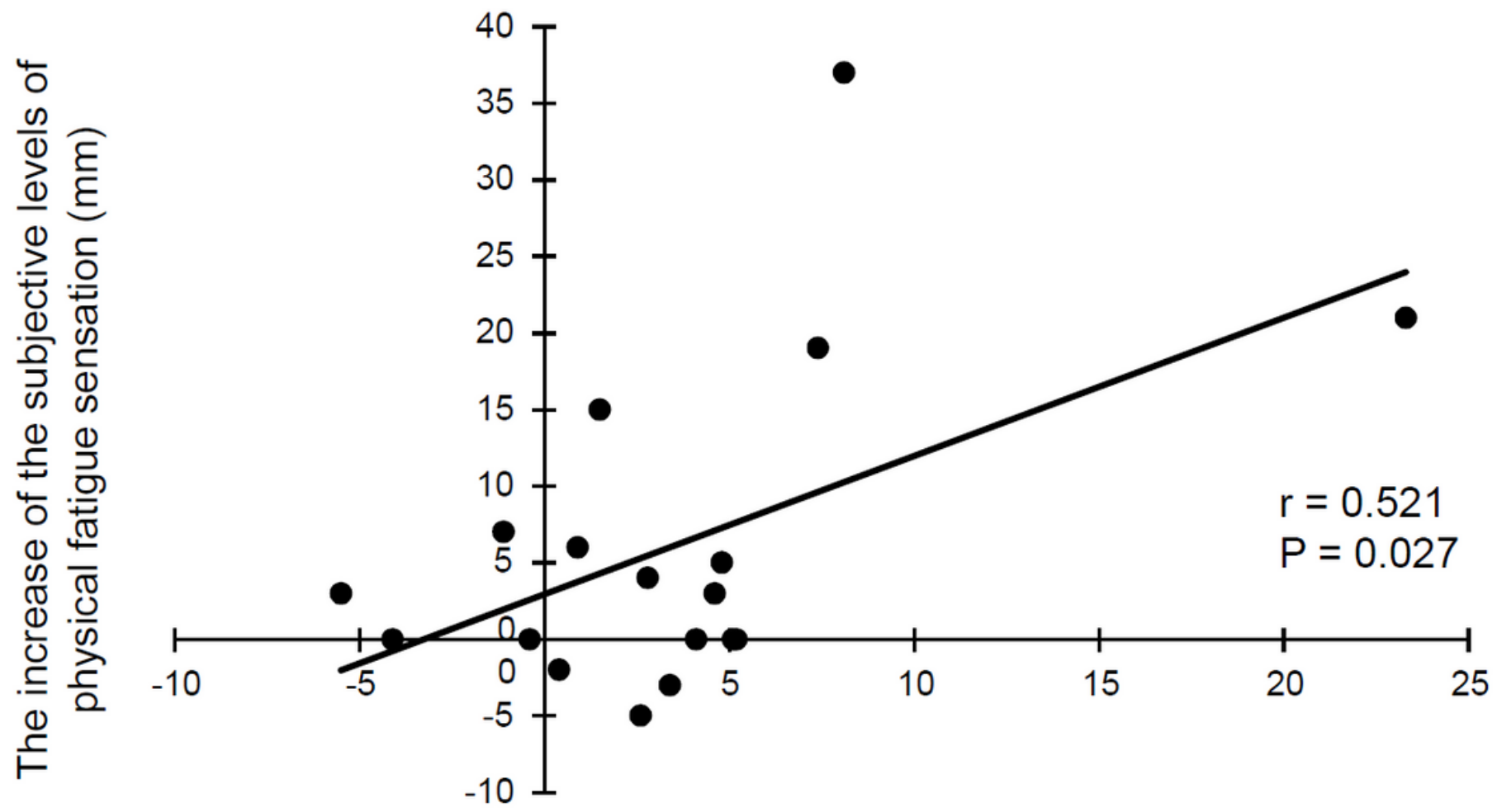

The decrease of the handgrip strength $(\mathrm{mm})$

Figure 4

Relationship between increased subjective level of physical fatigue sensation in the target condition and decreased handgrip strength in the target condition The linear regression line, Pearson's correlation coefficient, and $P$ values are shown. 


\section{Target condition > Control condition}

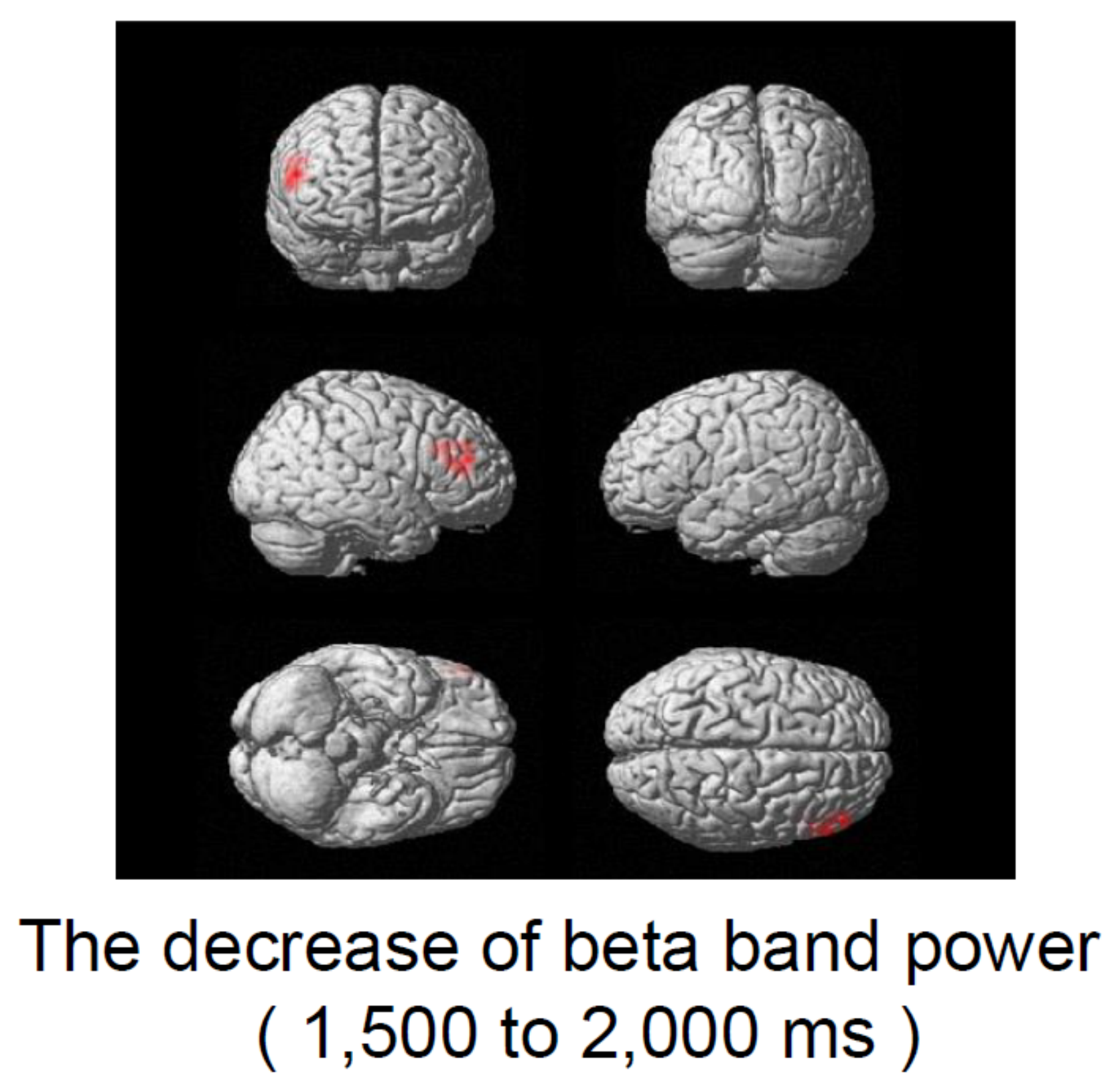

Figure 5

Statistical parametric map of the brain region in which the level of decreased beta band $(13-25 \mathrm{~Hz})$ power was higher in the target condition than in the control condition on the second day Random-effects analysis of 16 participants, $\mathrm{P}<0.05$, within BA 46 with small volume collection. 

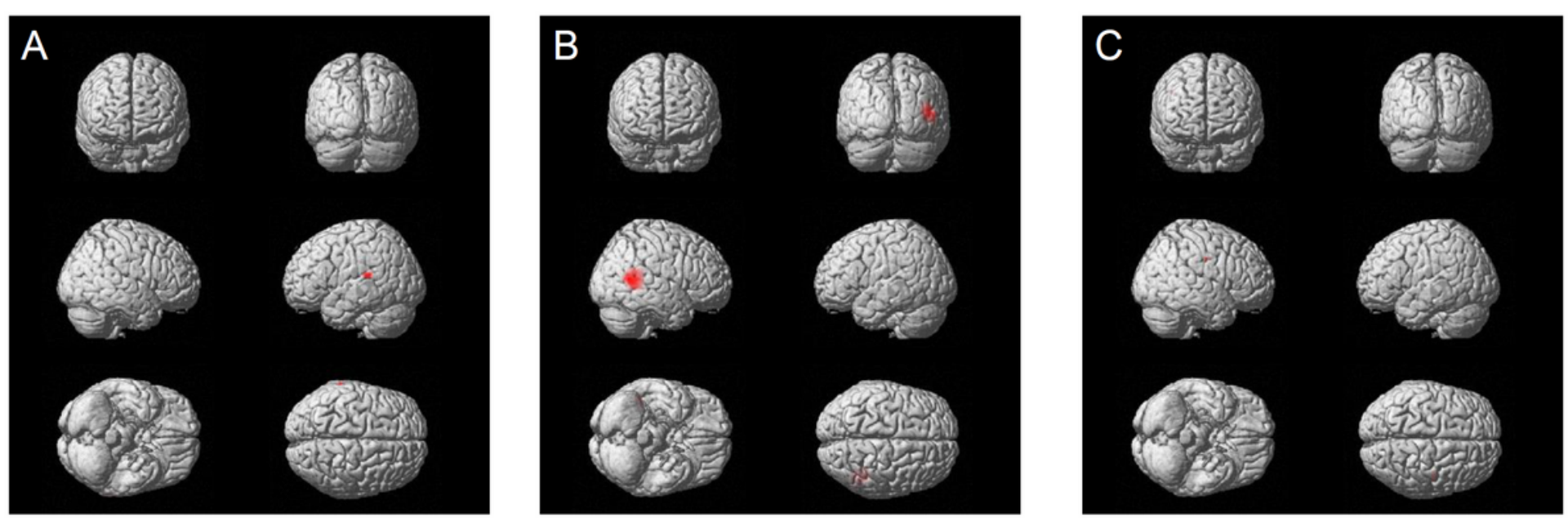

\section{Figure 6}

Statistical parametric map of the brain regions in which increased subjective levels of physical fatigue sensation in the target condition relative to that in the control condition correlated with alterations in oscillatory brain activity Brain regions showing increased subjective levels of physical fatigue sensation in the target condition relative to those in the control condition correlate negatively with increased theta band power (A), positively with increased gamma band power (B), and positively with decreased alpha band power (C). Random-effects analysis of 16 participants, $P<0.05$, with family-wise error correction for the entire search volume (whole brain). 